\title{
Seleção de cor dentária: Uma análise clínica dos métodos, técnicas e desafios cromáticos na odontologia restauradora
}

\author{
Dental color selection: A clinical analysis of methods, techniques and chromatic challenges in \\ restoring dentistry \\ Selección de color dental: Un análisis clínico de los métodos, técnicas y desafíos cromáticos en la \\ odontología restauradora
}

Recebido: 02/05/2021 | Revisado: 08/05/2021 | Aceito: 07/05/2021 | Publicado: 22/05/2021

\author{
Layla Narrely Santos Alves \\ ORCID: https://orcid.org/0000-0002-7497-3361 \\ Universidade Estadual da Paraíba, Brasil \\ E-mail: narrelylayla@gmail.com \\ Brenno Anderson Santiago Dias \\ ORCID: https://orcid.org/0000-0002-1047-3210 \\ Universidade Estadual da Paraíba, Brasil \\ E-mail: brennosantiagod@gmail.com \\ Francielly de Lemos Medeiros \\ ORCID: https://orcid.org/0000-0002-8672-7731 \\ Universidade Estadual da Paraíba, Brasil \\ E-mail: franciellylemos12@gmail.com \\ Jefferson Lucas Mendes \\ ORCID: https://orcid.org/0000-0003-0379-4101 \\ Universidade Estadual da Paraíba, Brasil \\ E-mail: jefflucasmendes@gmail.com \\ Geovanna Caroline Brito da Silva \\ ORCID: https://orcid.org/0000-0002-8943-5638 \\ Universidade Estadual da Paraíba, Brasil \\ E-mail: geovannacarolineb@gmail.com \\ Marcelo Gadelha Vasconcelos \\ ORCID: https://orcid.org/0000-0003-0396-553X \\ Universidade Estadual da Paraíba, Brasil \\ E-mail: marcelo.vasconcelos@yahoo.com.br \\ Rodrigo Gadelha Vasconcelos \\ ORCID: https://orcid.org/0000-0002-7890-8866 \\ Universidade Estadual da Paraíba, Brasil \\ E-mail: rodrigogadelhavasconcelos@yahoo.com.br
}

\begin{abstract}
Resumo
Introdução: Existe uma crescente demanda por restaurações estéticas que reproduzam com naturalidade as características da estrutura dentária. Para isso, é necessário que o cirurgião dentista compreenda o mimetismo dos dentes naturais, sobretudo sua cor, para obter restaurações estéticas satisfatórias. Objetivos: objetivou-se discutir acerca das etapas e do procedimento clínico para uma correta seleção de cor dentária, a fim de obter excelência nas restaurações e proporcionar à comunidade acadêmica conteúdo aprofundado sobre a temática. Materiais e Métodos: Foi realizada uma busca bibliográfica nas seguintes bases de pesquisa online: PUBMED/MEDLINE, LILACS, BBO, Scielo, Google Acadêmico, através do rastreio de artigos relevantes publicados entre o período de 2000 a 2020 . Foram utilizados os seguintes descritores para a pesquisa: Compomers (Compômeros), Color Selection (Seleção de cor), Tooth (Dente). Foi utilizado também o sistema de formulário avançado “AND”. Resultados: Os estudos obtidos com a pesquisa demonstram que a seleção cromática do dente é complexa e deve ser feita com embasamento teórico e prático. Na área da dentística estética a cor é dividida em três dimensões: matiz, croma e valor. Propriedades que influenciam diretamente na observação e seleção da cor. Ademais, a restauração deve reproduzir as características translúcidas do esmalte e opalescentes da dentina. Conclusão: A seleção de cor é um dos mais importantes passos em restaurações de dentes anteriores. Logo, é fundamental a experiência clínica e o estudo sobre a escolha da cor dentes, como também uma constante atualização do assunto, para obter restaurações sutis e imperceptíveis.
\end{abstract}

Palavras-chave: Cor; Estética dentária; Seleção de cor.

\footnotetext{
Abstract

Introduction: There is a growing demand for aesthetic restorations that naturally reproduce the characteristics of the dental structure. For this, it is necessary that the dentist understands the mimicry of natural teeth, especially their color,
} 
to obtain satisfactory aesthetic restorations. Objectives: Thus, the objective was to discuss about the steps and the clinical procedure for a correct selection of tooth color, in order to obtain excellence in restorations and provide the academic community with in-depth content on the subject. Materials and Methods: A bibliographic search was carried out on the following online research bases: PUBMED / MEDLINE, LILACS, BBO, Scielo, Google Scholar, through the screening of relevant articles published between 2000 and 2020. The following descriptors were used for the search: Composite Resins, Compomers, Color Selection, Tooth (Tooth). Results: The studies obtained with the research demonstrate that the chromatic selection of the tooth is complex and should be done with a theoretical and practical basis. In the field of cosmetic dentistry, color is divided into three dimensions: hue, chroma and value. Properties that directly influence color observation and selection. In addition, the restoration must reproduce the translucent enamel and opalescent characteristics of dentin. Conclusion: Color selection is one of the most important steps in anterior tooth restorations. Therefore, clinical experience and study on the choice of teeth color is essential, as well as a constant updating of the subject, in order to obtain subtle and imperceptible restorations.

Keywords: Color; Dental aesthetics; Color selection.

\section{Resumen}

Introducción: Existe una demanda creciente de restauraciones estéticas que reproduzcan de forma natural las características de la estructura dental. Para ello, es necesario que el cirujano dental entienda el mimetismo de los dientes naturales, especialmente su color, para obtener restauraciones estéticas satisfactorias. Objetivos: el objetivo fue discutir los pasos y el procedimiento clínico para la correcta selección del color del diente, con el fin de obtener la excelencia en las restauraciones y brindar a la comunidad académica un contenido profundo sobre el tema. Materiales y Métodos: Se realizó una búsqueda bibliográfica en las siguientes bases de investigación online: PUBMED / MEDLINE, LILACS, BBO, Scielo, Google Scholar, mediante la selección de artículos relevantes publicados entre 2000 y 2020. Para la búsqueda se utilizaron los siguientes descriptores : Compómeros, Selección de color, Diente (Diente). También se utilizó el sistema de formulario avanzado "Y". Resultados: Los estudios obtenidos con la investigación demuestran que la selección cromática del diente es compleja y debe realizarse con una base teórica y práctica. En el campo de la odontología cosmética, el color se divide en tres dimensiones: tono, croma y valor. Propiedades que influyen directamente en la observación y selección del color. Además, la restauración debe reproducir el esmalte translúcido y las características opalescentes de la dentina. Conclusión: La selección del color es uno de los pasos más importantes en las restauraciones de dientes anteriores. Por ello, la experiencia clínica y el estudio sobre la elección del color de los dientes son fundamentales, así como una actualización constante del tema, para obtener restauraciones sutiles e imperceptibles.

Palabras clave: Color; Estética dental; Selección de colores.

\section{Introduçãa}

A odontologia restauradora objetiva produzir restaurações imperceptíveis, sejam elas diretas ou indiretas. Um dos seus maiores desafios é a seleção de cores, visto que é um processo complexo mediado por inúmeros fatores que o cirurgiãodentista deve conhecer e ser capaz de realizar (Lucena et al., 2016). O crescente aumento da exigência estética do sorriso pelos indivíduos tem motivado os cirurgiões-dentistas a realizarem procedimentos restauradores com excelência e domínio. Desse modo, para a seleção de cor do dente deve-se ter uma atenção especial, a fim de atender aos desejos dos pacientes (Baratieri et al., 2013; Lucena et al., 2016; Ribeiro, 2019; Valenzuela-Aránguiz, 2016).

A cor é uma sensação psicofísica no qual se verifica a percepção visual da luz refletida por um objeto, que pode ser influenciada pelo observador, a fonte de luz e pelo objeto (Jain et al., 2016). Se a fonte de luz mudar, a luz refletida pelo objeto também mudará e uma nova cor será percebida. Sendo assim, a fonte de luz interfere diretamente na sensação da cor (Ribeiro, 2019; Valenzuela-Aránguiz, 2016).

As propriedades ópticas dos dentes se dão pela combinação dos efeitos da coloração extrínseca e intrínseca, como pela composição do esmalte e da dentina. O esmalte possui estrutura altamente mineralizada e pouco conteúdo orgânico, conferindo propriedades de transparência e opalescência (Ribeiro, 2019). Em contrapartida, a dentina contém maior quantidade de conteúdo orgânico e menos mineral, o que a torna menos translúcida, permitindo maior reflexão da luz, sendo responsável pela cor que se percebe (Ribeiro, 2019; Valenzuela-Aránguiz, 2016).

Assim, a seleção da cor é uma etapa subjetiva, pois existem algumas variáveis que podem interferir na seleção de cores, como a textura da superfície do dente, desidratação do dente, escala de cores, iluminação direta e indireta, diferenças de 
gênero, idade, fadiga visual do operador, além de distúrbios da visão à exemplo, o daltonismo (Bernardes, 2017; Junior, 2006; Lucena et al., 2016; Valenzuela-Aránguiz, 2016).

Dessa forma, esta etapa se caracteriza como uma das mais importantes nas restaurações dos dentes, dado que quando a cor não é escolhida corretamente leva a insatisfação e descontentamento do paciente (Reus, 2017). Portanto, o cirurgiãodentista deve ter seus conhecimentos aprofundados acerca da cor, luz e características das estruturas dos dentes naturais, além dos materiais restauradores, como as resinas. Ademais, deve saber como reconstruir adequadamente a anatomia dentária (Reus, 2017; Sousa, 2017).

Sendo assim, diante da relevância clínica do tema, o intuito desse artigo é trazer para a comunidade acadêmica mais informações sobre a seleção de cores na odontologia e analisar, em melhor compreensão, dicas clínicas para obter resultados estéticos satisfatórios.

\section{Materiais e Métodos}

O presente estudo constitui uma revisão da literatura do tipo narrativa realizada nas bases de dados eletrônicos: PubMed, LILACS, MEDLINE, Scielo, BBO, através da busca de artigos relevantes publicados entre o período de 2000 a 2020 relacionados com a temática da seleção de cor para as reconstruções dentárias. Os artigos foram selecionados usando os descritores encontrados no DECs, os quais foram: Composite Resins (Resina Composta), Compomers (Compômeros), Color Selection (Seleção de cor), Tooth (Dente). Foi utilizado também o sistema de formulário avançado "AND" para filtragem dos artigos relacionados ao tema. Os resultados obtidos através da pesquisa foram avaliados e classificados em elegíveis, que são os estudos que apresentaram relevância e tinham possibilidade de ser incluídos na revisão, e não elegíveis, ou seja, aqueles estudos sem possibilidade de inclusão na revisão. Ademais, para a seleção dos artigos foi considerado a disponibilidade do texto integral do estudo, clareza no detalhamento metodológico utilizado, artigos escritos em inglês, espanhol ou português e aqueles que se enquadravam no enfoque do trabalho. Além disso, foram incluídos livros referentes à temática estudada.

\section{Revisão de Literatura}

Segundo Munsell, um pintor americano que remonta ao ano 1898, a cor possui três dimensões que correspondem à matiz, croma e valor, com isso as cores podem ser expressas por suas interações (Lucena et al., 2016; Melo et al., 2020; Vanini, 2011). A matiz corresponde ao nome real da cor, pode ser interpretada como a cor base do dente ou tonalidade, é especificada como o intervalo dominante de comprimentos de onda no espectro visível que produz a cor percebida. Na escala Vita Classic Shade Guide ${ }^{\circledR}$ (VITA Zahnfabrik, Bad Säckingen, Alemanha), a matiz é representada pelas letras A, B, C e D. Sendo que o matiz A tem dominância nas cores vermelho-marrom, o B nas cores laranja-amarelo, o C nas cores verde-cinza e por último, o D tem dominância rosa-cinza (Baratieri et al., 2013; Ferraris, 2015; Lucena et al., 2016; Pereira, 2017; Sousa, 2019).

O croma, por sua vez, é definido como a saturação ou intensidade da matiz (Vanini, 2011). Varia com a quantidade de pigmento incorporado numa matiz e determina a intensidade da cor, isto é, se forte ou fraca. O croma e o valor estão inversamente proporcionais visto que quando o croma é aumentado, o valor é diminuído. Na escala Vita Classic ${ }^{\circledR}$ (VITA Zahnfabrik, Bad Säckingen, Alemanha) números mais altos na guia de cores representam um aumento de croma, assim as letras representam o matiz e os números o croma. É importante enfatizar que o matiz e o croma estão presentes na dentina, pois são propriedades dessa estrutura (Lucena et al., 2016; Melo et al., 2020; Pereira, 2017; Sikri, 2010; Sousa, 2019).

O valor, por fim, é determinado como a luminosidade do objeto. A luminosidade de qualquer objeto é uma consequência direta da quantidade de energia de luz que o objeto reflete ou transmite (Lucena et al., 2016; Vanini, 2011). O valor pode ser descrito como uma escala de cinza, de branco a preto. Objetos brilhantes possuem alto valor, e por isso têm 
quantidades mais baixas de cor cinza e os objetos de baixo valor, mais escuros, têm quantidades maiores de cinza (Bhatia, 2013; Lucena et al., 2016; Pereira, 2017; Sousa, 2019).

Dentre todas as dimensões, na odontologia, o valor é a dimensão mais importante para a seleção cromática do dente, já que é essa a qualidade que define o claro e o escuro, e, segundo algumas metodologias, deve ser a primeira dimensão a ser determinada visto que as discrepâncias de valor são mais facilmente identificáveis esteticamente do que as discrepâncias de croma e matiz (Amaral, 2006; Derbabian et al., 2001; Lucena et al., 2016; Magne e Belser, 2002; Melo et al., 2020).

De acordo com Hirata (2017) o olho humano é mais sensível a mudanças do valor e ao croma, sendo assim, as escalas de cor que são baseadas no valor são mais precisas. Ao comparar, por exemplo, um tom B1 com um A1, é difícil avaliar qual a amostra que apresenta uma cor mais laranja ou mais amarela, porém, é relativamente mais fácil identificar qual a amostra se apresenta mais intensa/brilhante, no qual seria o valor (Hirata, 2017; Gurel, 2003).

Além disso, um valor muito elevado produz uma restauração com aspecto mais artificial, podendo ser percebida com facilidade, já um valor reduzido tira a vida da restauração, mesmo que o matiz e o croma estejam corretos (Tabela 1) (Lucena et al., 2016).

Tabela 1 - Características das restaurações com baixo valor e alto valor, respectivamente.

\begin{tabular}{|c|c|}
\hline \multicolumn{2}{|c|}{ VALOR } \\
\hline BAIXO VALOR & ALTO VALOR \\
\hline Menos luminoso & Mais luminoso \\
\hline Mais escuro & Mais claro \\
\hline Mais cinza & Mais branco \\
\hline Mais translúcido & Mais leitoso \\
\hline Menor reflexão & Menor saturação \\
\hline Maior saturação & Menor croma \\
\hline Maior croma &
\end{tabular}

Fonte: Autores.

Além das propriedades ópticas objetivas, o dente também apresenta os elementos ópticos subjetivos, que influenciam na aparência final de uma restauração, como a translucidez, opalescência e fluorescência. Uma reprodução incorreta de algumas dessas características afetam o resultado estético final e produz uma aparência artificial, resultando em uma restauração mal sucedida (Reus, 2017; Sousa, 2019).

A translucidez pode ser definida pela quantidade de luz que é transmitida através de determinado material, é caracterizada por ser um gradiente entre a completa transparência e a opacificação, em que se tem a reflexão completa da luz. Esta é uma propriedade que pode ser bastante difícil quantificar em dentição, uma vez que varia de indivíduo para indivíduo e possui variados graus de translucidez (Villarroel et al., 2011). Apesar da dentina ser mais opaca que o esmalte, sua translucidez aumenta com a idade. Nos dentes, o terço incisal é a região mais translúcida, pois a espessura de dentina é menor que a do terço médio e cervical (Villarroel et al., 2011; Radaelli, 2013; Reus, 2017; Sousa, 2019). Dentes mais jovens tendem a apresentar pouca translucidez, por possuírem maior quantidade de dentina, já dentes mais velhos são mais translúcidos pelo desgaste natural do dente (Villarroel et al., 2011). 
O esmalte apresenta características estruturais diferentes da dentina, pois é uma estrutura prismática altamente mineralizada, um baixo conteúdo orgânico e uma pequena quantidade de água, além disso, possui uma transmissão de luz maior que a dentina (Radaelli, 2013; Villarroel et al., 2011). A dentina, em contrapartida, tem menos conteúdo mineral, uma estrutura tubular orgânica, um alto teor de água e é menos translúcida. Dessa forma, a maior parte das resinas compostas que são utilizadas para a reconstrução da dentina, possuem características de baixa translucidez, enquanto que as usadas para o esmalte são altamente translúcidas. É importante conhecer e saber qual a espessura necessária da resina para obtermos uma translucidez adequada para esmalte e dentina (Radaelli, 2013; Reus, 2017; Schmeling et al., 2012).

A opalescência ocorre quando um material parece ser de uma cor quando a luz é refletida e de outra cor quando a mesma é transmitida através dele (Amaral, 2006; Magne e Belser, 2002; Radaelli, 2013). É uma característica do esmalte dentário relacionada a sua capacidade de transmitir seletivamente ondas longas do espectro (alaranjadas) ao mesmo tempo que reflete as ondas curtas (azuis e violetas), ou seja, quando a luz alcança o esmalte dentário o comprimento de onda azul retorna para a visão do operador e o comprimento de onda laranja atravessa o dente (Villarroel et al., 2011). Quando as ondas curtas do espectro atinge o esmalte cria as tonalidades de azul claro e cinza que se tornam claramente visíveis ao nível da borda incisal (Villarroel et al., 2011; Radaelli, 2013; Reus, 2017; Sousa, 2019).

Concomitantemente, em materiais opalescentes como o dente, é possível observar também a contra-opalescência, que é um efeito no qual a luz penetra um material opalescente e é refletida dentro do próprio material (Andrade e Couto, 2017). Este fenômeno se caracteriza por apresentar um brilho amarelo-avermelhado na região incisal dos mamelos. A região incisal dos dentes, por se tratar de uma área com menor quantidade de dentina e, portanto, mais transparente, possibilita a visualização das características de opalescência e contra-opalescência (Pepelascov et al., 2018).

Em contrapartida, a fluorescência é uma propriedade da dentina que confere aos dentes naturais o aspecto de vitalidade e luminosidade. Este fenômeno ocorre pela absorção de raios ultravioleta, emitidos pela luz solar, ou por qualquer outra iluminação ultravioleta que excite os componentes fotossensíveis presentes no esmalte e na dentina (Lucena et al., 2016). A fluorescência deve ser reproduzida nas restaurações estéticas para obter uma aparência natural e de vitalidade, pois esta propriedade contribui para que os dentes pareçam mais brancos e claros na luz do dia, luz negra ou luzes artificiais, além de conferir profundidade óptica e vitalidade (Roesner, 2007; Reus, 2017; Sousa, 2019).

A seleção de cor é a capacidade do operador de conseguir correlacionar as cores reais da dentição natural com as da resina composta. Contudo, essa seleção exige conhecimento e concentração, pois diversos fatores, como a fonte de luz, o ambiente e as escalas de cor, podem influenciar na precisão da cor. Dessa forma, o cirurgião-dentista deve dispor das melhores técnicas para selecionar a cor durante a reconstrução de um dente (Pereira, 2017; Reus, 2017; Sousa, 2019).

\subsection{Técnicas para avaliação da cor do dente}

A reprodução da cor dos dentes é um grande desafio na área da estética dentária. Há diversos métodos para a determinação da cor na Odontologia, entretanto há aqueles mais usados na prática clínica. Dentre eles, podemos realizar uma análise comparativa entre uma escala de cores que são compostas por amostras representativas das médias das cores presentes na dentição humana, como a VITA Classic ${ }^{\circledR}$ e VITA 3D-Master Shade Guide ${ }^{\circledR}$ (VITA Zahnfabrik, Bad Säckingen, Alemanha). (Baratieri, 2013; Lucena et al., 2016; Reus, 2017; Sousa, 2019)

A escala de cores VITA Classic $^{\circledR}$ é baseada no matiz e croma, em que o matiz possui as nuances de A, B, C e D, os quais são, respectivamente, castanho, amarelo, cinza e vermelho; já o croma é dividido em 4 graus, variando de 1 a 4 . Desta forma, o matiz A apresenta cinco intensidades cromáticas (A1, A2, A3, A3,5, A4), o matiz B e o C, apresentam-se com quatro (B1, B2, B3 e B4; C1, C2, C3 e C4), por último, o matiz D apresenta apenas três (D2, D3 e D4). Podem ser ordenadas pelo croma, seguindo a ordem A, B, C e D, ou organizadas em ordem decrescente de valor: B1, A1, B2, D2, A2, C1, C2, D4, A3, 
D3, B3, A3,5, B4, C3, A4 e C4 para facilitar a seleção de cores com base no valor (Baratieri, 2013; Igiel et al., 2016; Ferreira, 2017; Reus, 2017; Sousa, 2019).

Segundo Radaelli et al. (2013) o olho humano é mais sensível a dimensão do valor, isso acontece devido a um maior número de bastonetes (células fotorreceptoras envolvidas na transdução de luz monocromática em sinais nervosos para o cérebro através do nervo óptico) do que cones (células fotorreceptoras que detectam diferentes cores) na retina humana. Logo, um erro na avaliação do valor terá grande impacto sobre a percepção da cor final.

Além disso, há uma concordância nos trabalhos acadêmicos sobre a importância da escala de cores ser organizada pelo valor, visto que se o brilho está correto os dentes terão um aspecto mais natural e harmônico e as pequenas diferenças entre matiz e croma não serão percebidas. Ao contrário, se os dentes possuem valores diferentes e incorretos poderão ser notados com facilidade. Portanto, tal disposição da escala de cores, baseada no valor, facilita a correta seleção, ao direcionar o início da escolha para a seleção do valor do dente (Young et al., 1994; Amaral, 2006; Radaelli et al., 2013).

Por este motivo, se torna uma grande desvantagem da escala VITA Classic $^{\circledR}$ ser baseada apenas no matiz e croma, não contendo o valor, visto que facilita e é essencial para uma correta seleção de cor (Vanini e Mangani, 2001). Somado a isso, as escalas são geralmente mais amarelas do que a dentição natural e oferecem poucas opções de cores claras para dentes naturais de altos valores, como também resulta em restaurações planas e sem luminosidade (Vanini e Mangani, 2001; Amaral, 2006; Sousa, 2019).

Ademais, a escala VITA Classic ${ }^{\circledR}$ não apresenta tonalidades de cores mais escuras como as dos dentes de pacientes idosos, como também não abrange a coloração pós-clareamento dos dentes. Não dispõe de opções para diferentes tipos de translucidez e as diversas marcas de resina composta apresentam diferenças de cores e opacidade significativas quando comparadas a seus correspondentes nessa escala. Para se adaptar a tais necessidades, alguns autores sugeriram que as amostras da escala VITA Classic $^{\circledR}$ fossem realocadas de acordo com o valor: B1, A1, D2, A2, B2, C1, C2, D3, D4, A3, B3, A3, 5, B4, A4, C3, C4 (Amaral, 2006; Ferreira, 2020; Sousa, 2019).

Para reduzir essa lacuna, foi criada a escala VITA 3D-Master Shade System ${ }^{\circledR}$, que visa ampliar a cobertura do espaço cromático dos dentes e organiza mais uniformemente as cores em três dimensões, ou seja, matiz, croma e valor (Amaral, 2006; Lucena, 2016).

A escala VITA 3D-Master possui 26 cores e as divide em 5 grupos segundo a luminosidade da cor (valor) visto que é considerada a dimensão mais importante. Nos grupos de valor 2, 3 e 4 existem três amostras cromáticas que representam o matiz e são divididas por letras, M (médio), L (amarelo) e R (vermelho). Ao fazer uso dessa escala para selecionar a cor do dente, devemos iniciar definindo o grupo que possui o valor mais semelhante ao respectivo dente. Realiza-se a seleção do croma, comparando as diferentes saturações na coluna central do grupo e em seguida escolhe o matiz (Baratieri; Dario et al., 2016; Reus, 2017; Schmeling et al., 2017; Sikri, 2010).

Iniciamos a seleção com a VITA 3D-Master ${ }^{\circledR}$ (figura 1) posicionando a escala a uma certa distância da boca aberta do paciente e escolhendo, primeiramente, o valor (nível de claridade). Determinamos o grupo 1, 2, 3, 4 ou 5 escolhido. Escolher o que mais se aproxima da claridade (valor) do dente em questão. Nos grupos de valor 2, 3 e 4 existem três colunas de amostras cromáticas com as letras M (médio), L (amarelo) e R (vermelho). Inicialmente, a seleção do croma deve ser realizada na coluna da letra M. Estas letras representam o matiz, isto é, o último passo da seleção de cor. Logo, confirma a matiz, avaliando se o dente natural está mais avermelhado (R) ou amarelado (L) do que a cor selecionada (Bernardes, 2017; Schmeling et al., 2017; Bottino, Faria, Valandro, 2009). 
Figura 1 - Escala VITA 3D-Master (VITA Zahnfabrik, Bad Säckingen, Alemanha). Três passos $\left(1^{\circ}\right.$ valor; $2^{\circ}$ croma e $3^{\circ}$ matiz) a serem seguidos para a seleção de cores utilizando a escala VITA 3D-Master ${ }^{\circledR}$. Primeiro é realizado a definição do nível de claridade (1-5) pelas tonalidades médias (M), escolhendo o que mais se aproxima do valor do dente em questão. Em seguida é realizada a seleção do croma dentro do grupo de valor escolhido. Por fim, verifica se o dente natural apresenta uma tonalidade mais "amarelada" (L) ou "avermelhada" (R) do que amostra de cor do grupo "médio" (M) selecionada no $2^{\circ}$ passo.

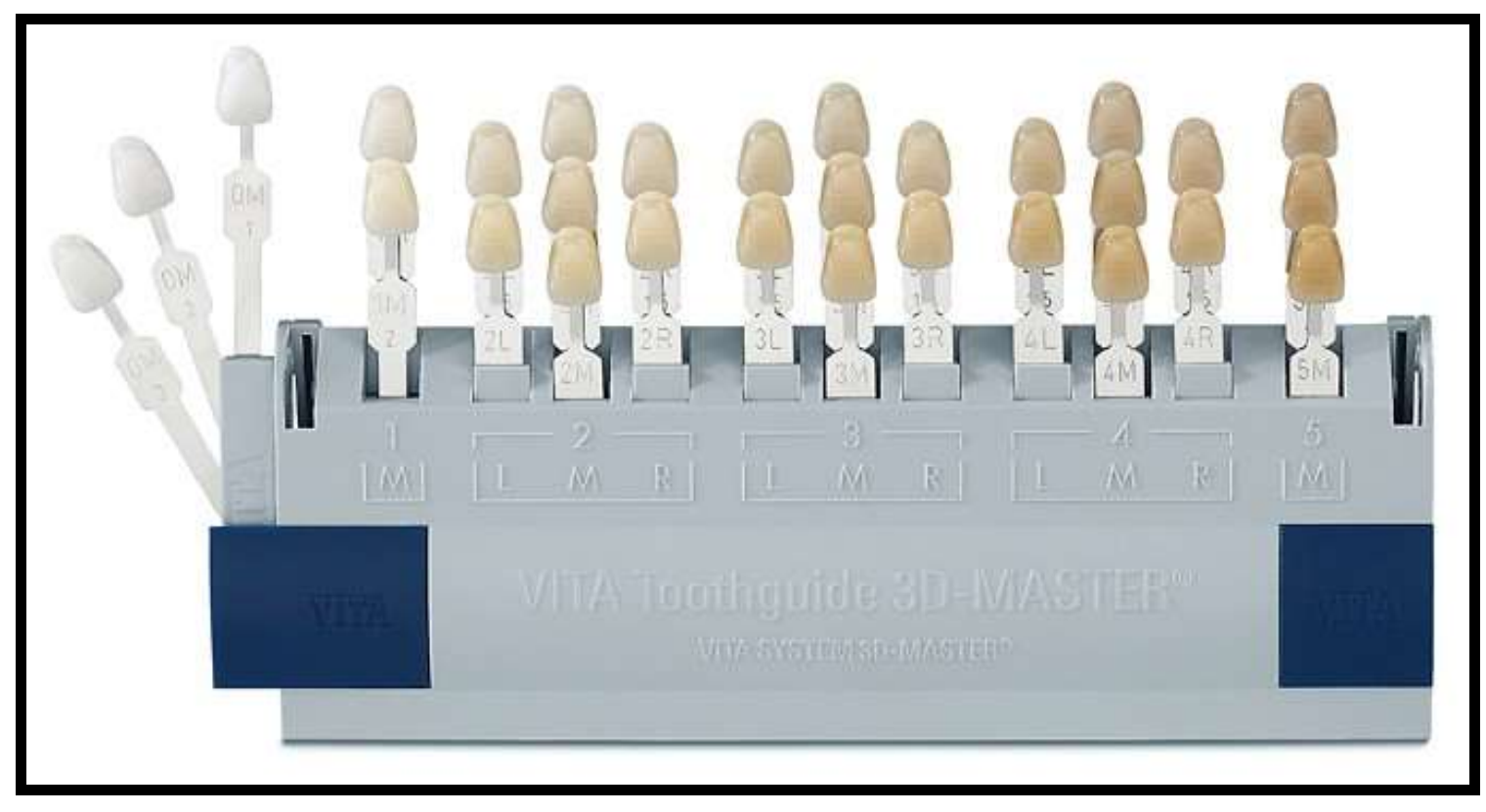

Fonte: Sousa, 2019.

A maior limitação das escalas de cores, de modo geral, deve-se ao fato de grande parte dos fabricantes de produtos odontológicos não a utilizarem como padrão de cores no desenvolvimento de seus produtos, principalmente das resinas compostas diretas e indiretas (Lucena et al., 2016).

Por muito tempo a escala Vita ${ }^{\circledR}$ foi tida como referência para determinação da cor dentária. Porém, ao longo dos anos, foram encontradas complicações em relação ao seu uso, à exemplo, o tempo desperdiçado ao tentar obter uma amostra cromática ideal, que na verdade não existia, mas sim uma aproximação. Ainda hoje, a escala Vita ${ }^{\circledR}$ é utilizada, o que aumenta as chances de erro já que não é possível vincular as atuais resinas de cada fabricante as amostras de cores presentes escala Vita $^{\circledR}$ (Dantas e Florez, 2011; Manauta e Salat, 2013).

Alguns estudos concluíram que a escala 3D-Master ${ }^{\circledR}$ apresenta cores distribuídas mais uniformemente e abrangente no espaço cromático, fornecendo uma melhor precisão e facilidade na escolha da cor comparada a escala VITA Classical ${ }^{\circledR}$ (PARAVINA, 2009). Gasparik et al. (2015) mostram que quando as guias de cores foram comparadas, os resultados evidenciaram que a escala VITA Classical $^{\circledR}$ conduziu aos melhores escores de comparação em relação ao $3 D$ Master ${ }^{\circledR}$, contrariando o estudo anterior.

Em contrapartida, para Negahdari et al. (2016) há diferenças significativas entre estes dois sistemas de guias de cores, com o sistema 3D Master $^{\circledR}$ (Vita Zahnfabrik, Germany), exibindo maior grau de reprodução em comparação com o sistema VITA Classical $^{\circledR}$ (Vita Zahnfabrik, Germany).

Concomitantemente, em uma revisão de literatura de Pereira (2019), o método visual de seleção de cor a escala VITA 3 D-Master ${ }^{\circledR}$ mostrou-se melhor que o guia de cores VITA Classical ${ }^{\circledR}$. Para Marcucci (2003) a seleção de cor utilizando a escala VITA 3D-Master ${ }^{\circledR}$ possui mais vantagens, pois se baseia no princípio tridimensional da cor, possui uma distribuição equidistante no espaço cromático da dentição natural, além de preconizar o valor como primeira escolha. Entretanto, ao 
realizar um questionário com 60 cirurgiões-dentistas sobre o uso do guia de cores VITA, Rodrigues (2007) mostra que todos os dentistas utilizaram o método de seleção usando o guia Vita, em que 51,7\% usavam apenas o VITA guide, enquanto $6,7 \%$ usaram apenas o guia de cores $3 D$-Master ${ }^{\circledR}$.

Em suma, é importante enfatizar que as escalas não conseguem reproduzir perfeitamente a cor dos dentes, apesar de existir diversas escalas no mercado odontológico, visto que possuem limitações cromáticas que dificultam a realização de restaurações que alcancem ao máximo a reprodução do dente natural (Ferreira, 2017).

Com o passar dos anos, houve a introdução de aparelhos eletrônicos que realizam a determinação da cor dentária, são os chamados espectrofotômetros, colorímetros. Estes agem emitindo luz e avaliando o espectro refletido pelos dentes, identificando as cores presentes. O espectrofotômetro é um instrumento preciso para selecionar a cor, pois identificam quando é incidida uma fonte de luz no objeto. Eles conseguem detectar pequenas diferenças entre as cores que não são captadas pelo olho humano, contribuindo para a diminuição da subjetividade e dos erros no procedimento e aumentando, consequentemente, a objetividade (Al-hammad et al., 2010; Ferreira, 2017; Lucena et al., 2016). Existem vários no mercado, como o Crystaleye ${ }^{\circledR}$ (Olympus, Tokyo, Japan), o Vita Easy Shade Compact $^{\circledR}$ (VITA Zahnfabrik, Bad Säckingen, Alemanha), Spectro Shade Micro ${ }^{\circledR}$ (MHT Optic Research, Niederhasli, Switezerland), geralmente são portáteis e sem fios (Chu et al., 2010).

Os colorímetros, em comparação, são mais simples e de baixo custo, desenvolvidos para medir a cor baseado em três eixos cromáticos ou estímulos, utilizando um filtro simulador do olho humano. São mais inferiores em relação ao espectrofotômetro por serem menos precisos na seleção da cor, além de registrarem o espectro de reflexão (Baratieri, 2013; Lucena et al., 2016; Reus, 2017; Sousa, 2017).

É válido ressaltar as grandes vantagens desses métodos para determinar a cor de um dente, como a ausência do cansaço do profissional, padronização da iluminação e obtenção de ótimos resultados. Os possíveis erros acontecem por, dentre outras coisas, má calibração do aparelho (Kalantari et al., 2017; Schmeling et al., 2017).

Segundo Schmeling et al. (2017) e Kalantari et al. (2017), a escolha da cor é melhor desenvolvida com a técnica do espectrofotômetro, quando comparada ao método visual utilizando escalas de cores, concordando com a revisão de literatura realiza por Pereira (2019).

Existem também as câmaras digitais, que são instrumentos cada vez mais usados pelos cirurgiões dentistas pela sua facilidade de utilização e eficiência. Ao invés de focar a luz para realizar uma reação química, as câmaras digitais capturam as imagens usando dispositivos de carga emparelhada, que contêm milhões de elementos microscopicamente pequenos e sensíveis à luz (Kalantari et al., 2017). A maioria dos sensores utilizam um filtro que permite observar na luz as suas três cores primárias para obter uma imagem totalmente colorida. Um exemplo das câmaras são o ShadeRite Dental Vision System ${ }^{\circledR}$ e o ShadeScan ${ }^{\circledR}$. Entretanto, o uso das câmeras são métodos auxiliares que devem ser usados como complementares ao método visual, visto que o local ou a iluminação podem interferir na imagem, necessitando do olhar do operador (Agrawal, 2013; Kalantari et al., 2017; Sikri, 2010).

Outra técnica, bastante utilizada na prática clínica odontológica em restaurações diretas é a aplicação e fotoativação de pequenos incrementos de compósitos sobre a superfície do dente em questão. A principal vantagem desse método é por usar o próprio sistema de resina composta para selecionar a cor, como também é mais prático. Ao realizar essa técnica, indica-se aplicar incrementos de resina para esmalte, pois é este que determina a cor final do dente (Baratieri, 2013). É colocado um pequeno incremento de resina composta, aproximadamente $2 \mathrm{~mm}$ de espessura, junto ao terço cervical do dente, local onde se determina a cor da dentina. Em contrapartida, deve-se observar se a ponta da borda incisal tem maior ou menor valor para a escolha do valor (Lucena et al., 2016). 
Devido as limitações das escalas de cores comerciais, alguns autores adotam a técnica de produção da própria guia de cores. O profissional pode confeccionar sua própria escala de cor com a marca de resina que mais utiliza no dia a dia, facilitando assim o processo de seleção de cor (Paolone et al., 2014).

Pode-se realizar essa técnica através de dois métodos principais, por meio de um micrômetro, este é um dispositivo barato que incorpora um parafuso calibrado. Com isto, é possível obter espécimes redondos com espessura extremamente precisa, podendo sobrepor tanto a resina de dentina como a de esmalte. Contudo, esse método não fornece o formato da convexidade dos dentes naturais (Paolone et al., 2014).

Outro método para realizar essa técnica é através de moldes pré-fabricados, em que se tem porções positivas e negativas do molde. A parte negativa é a que reproduz o esmalte vestibular, feito de plástico semi-rígido, já a parte positiva exibe a forma do corpo dentinário e é feito de silicone transparente e macio. Para fazer a camada de esmalte são determinadas diferentes espessuras, como $0,2 \mathrm{~mm}$ de espessura no terço cervical, $0,5 \mathrm{~mm}$ no terço médio meio e $0,7 \mathrm{~mm}$ de espessura na porção incisal. Na confecção desse método pode utilizar resinas de esmalte e de dentina da mesma marca ou de marcas variadas (Paolone et al., 2014).

O mapa cromático do dente consiste em um meio de realce e comunicação, no qual todos os parâmetros que contribuem para a criação da cor no dente são refinados e anotados. Ou seja, são todas as informações mais detalhadas das características individuais dos dentes, como também das suas diferentes regiões (Costa, 2018; Lobato et al., 2019).

Assim, o mapa cromático possui diferentes expressões nos vários níveis anatômicos: os cromas mais altos estão no terço cervical, pois é a área com maior saturação devido o esmalte ser mais fino nesse local; já no terço médio os cromas são mais baixos e no terço incisal há apenas a translucidez do esmalte incisal. Logo, esta técnica permite o planejamento prévio para determinar a cor do dente nas restaurações, ao realizar esquematizações e combinações com as cores, facilitando o processo e evitando erros posteriores (Cardoso et al., 2006; Lobato et al., 2019; Tokumi, 2007).

Para muitos autores, a fotografia tem sido bastante utilizada como um meio para realizar o mapeamento cromático. Principalmente a tomada de fotografias quando analisadas em preto e branco, pois permite avaliar o valor do dente. Dessa forma, a primeira etapa do mapeamento cromático é a realização das fotografias dos dentes (de preferência um dente hígido análogo ao que será restaurado) e transferência para o Photoshop ${ }^{\circledR}$ (programa de edição de imagens). A partir daí utiliza-se filtros específicos do Photoshop ${ }^{\circledR}$ que evidenciem a visualização das nuances e detalhes cromáticos presentes nos dentes (Cardoso et al., 2006; Costa, 2018; Lobato et al., 2019; Tokumi, 2007).

\subsection{Principais variáveis que interferem na seleção da cor dentária}

Por ser uma análise subjetiva, conforme já mencionado, a seleção de cor se torna bastante difícil, causando muitas vezes nos profissionais frustrações e insucessos. Por vezes, essas falhas se devem à falta de domínio das variáveis que interferem durante a seleção e produz resultados insatisfatórios. Logo, a compreensão da cor e dos fatores que afetam esse procedimento, assim como a metodologia da combinação das cores deverão ser conhecidos e aplicados na prática clínica odontológica a fim de minimizar os erros durante a seleção da cor dentária (Amaral, 2006; Reus, 2017; Sousa, 2019; Lima et al., 2013).

De acordo com Amaral (2006), existem três categorias básicas que interferem na seleção da cor, são elas: as variáveis do objeto, da fonte de luz e do observador.

\subsubsection{Variáveis do objeto}

Além da translucidez, fluorescência e opalescência do dente, os quais são propriedades que, uma vez entendidas, auxiliam na obtenção correta da cor, os aspectos de textura e desidratação dentária influencia na escolha. As variáveis da 
textura do objeto podem produzir diferenças visíveis entre duas cores que, de outra forma, seriam idênticas (Amaral, 2006; Reus, 2017). A texturização superficial está intimamente ligada com a cor do dente, pois um dente jovem, por exemplo, proporciona uma maior reflexão de luz em diferentes direções por ter lobos e sulcos evidentes, transmitindo a sensação de um dente mais claro. Inversamente, dentes com menos texturização, ou seja, com superfície mais lisa, são aparentemente mais escuros (Lucena et al., 2016; Melo et al., 2020).

É importante considerar o tamanho e a forma do dente, pois são características que interferem nos ângulos de refração e reflexão da luz, influenciando na cor percebida do dente. Em relação a forma, o dente pode ser considerado quadrado, possuindo contornos paralelos; os ovóides, que apresentam linhas externas arredondadas com convergência para cervical e incisal e, por fim, os triangulares, tem ângulos incisais pronunciados e convergência para cervical. Em relação ao tamanho do dente o operador deve preservar o comprimento da coroa tanto em largura como em altura para manter o aspecto mais natural possível (Lucena et al., 2016).

A seleção de cor deve ser realizada em ambientes que tenham paredes neutras, de preferência de cor branca, devido a reflexão da luz nos objetos. Os dentes devem estar devidamente limpos e com a umidade natural da cavidade oral, pois a água possui um papel fundamental no resultado final da cor (Amaral, 2006; Lucena et al., 2016). O esmalte desidratado é menos translúcido, devido a taxa de refração da luz na água e no ar ser diferente, a secagem do esmalte faz com que o ar circunde os espaços interprismáticos, o que pode confundir o cirurgião-dentista a escolher uma resina mais clara e opaca do que a cor natural do dente (Reus, 2017; Nahsan et al., 2012).

Quando realizado o isolamento absoluto e feita a secagem dos dentes com jatos de ar, após 30 minutos as regiões desidratadas parecem com maior luminosidade do que quando umedecidas. Já no esmalte, as alterações de aparência aparecem em menos de 10 minutos após a secagem. Dessa maneira, é indicado que a seleção de cor seja feita antes do isolamento absoluto para ter a observação ideal da cor do dente e, ainda, com os dentes levemente hidratados (Amaral, 2006; Ottoboni et al., 2018; Lucena et al., 2016; Pereira, 2019). Em um estudo realizado por Jain, Bhat e Hegde (2017) com 42 dentistas e 74 estudantes de odontologia, eles observaram que $72,4 \%$ dos dentistas realizavam a seleção de cor antes de qualquer procedimento cirúrgico, visto que a cor dos dentes altera na medida que perdem a consistência durante o preparo.

\subsubsection{Variáveis da fonte de luz}

Segundo Barh et al. (2015) o fator que mais interfere na seleção visual da cor do dente é a iluminação, de modo que é fundamental o conhecimento correto da melhor fonte de luz para essa escolha. A fonte de iluminação utilizada durante a seleção da cor pode mudar a aparência da escala de cor e dos dentes naturais, a este fenômeno chamamos de metamerismo, onde duas amostras são iguais sob influência da mesma fonte luminosa e diferentes noutra. Para reduzir ou eliminar o metamerismo é importante utilizar mais do que uma fonte luminosa a partir de vários ângulos (Amaral, 2006; Reus, 2017; Sousa, 2019).

Com isso, diversos autores concordam que a luz natural, ou seja, a luz solar é considerada a ideal para uma correta seleção visual da cor. Esta deve englobar todo o espectro luminoso visível, manter-se constante e a uma temperatura de aproximadamente $5500 \mathrm{~K}$. Sendo essencial utilizá-la entre o período das 10 às 14 horas, pois é quando se tem as condições mais favoráveis para a determinação da cor (Amaral, 2006; Bahr et al., 2015; Rodrigues, 2007). A observação das cores não deve durar mais que 5 segundos a fim de evitar a fadiga dos cones dos olhos (Melo et al., 2020).

Existem três principais fontes de luz. A luz quente, na qual tem uma baixa temperatura, variando de $3000 \mathrm{~K}$ a $4000 \mathrm{~K}$, sendo incandescente e amarelada. A luz fria, que possui uma alta temperatura, está entre $5000 \mathrm{~K}$ a 6900K, porém é fluorescente, com um aspecto mais azulado. Por fim, tem a luz morna, de 4000K a 5000K. Dessa forma, quando não tem a disponibilidade da luz natural, em que é a melhor forma de selecionar a cor, é preferível o uso da luz fria, em detrimento da luz 
quente, pois engloba vários espectros de luz visível (Bahr et al., 2015; Rodrigues, 2007; Śmielecka e Dorocka-Bobkowska, 2020).

É válido mencionar que, durante a determinação da cor do dente, não se deve utilizar a luz do refletor do consultório odontológico nem permanecer com ele ligado, visto que a luz que predomina no refletor é a luz quente. Pode-se, no entanto, deixar a luz do ambiente predominar para facilitar a correta seleção (Amaral, 2006; Bahr et al., 2015).

\subsubsection{Variáveis do observador}

Algumas pesquisas sugerem que os observadores divergem muito na seleção da cor dos dentes. A percepção da cor pelo operador e a sua capacidade de distinguir duas cores quando comparadas lado a lado é influenciada pelas suas condições psicológicas e físicas. Esta deve ser feita por dentistas com uma visão cromática normal e os que têm deficiências na visão da cor devem ser auxiliados. Logo, a fadiga e o cansaço ocular contribuem com maiores chances de erros na seleção de cor, pois dificultam na diferenciação do matiz e croma do objeto (Amaral, 2006; Moodley et al., 2015; Reus, 2017).

Na pesquisa de Jain, Bhat e Hegde (2017) a maior parte dos indivíduos sentem a necessidade de um auxiliar durante a seleção de cores, pois é importante uma segunda opinião durante a determinação da cor. Além disso, a idade do operador é tida como influenciadora nesse processo, visto que com o passar dos anos a córnea tende a ficar mais amarelada o que dificulta a diferenciação entre o amarelo e o branco. No entanto esta característica só se apresenta clinicamente relevante após os 60 anos de idade (Ferreira, 2020).

Outro fator que tem sido pautado como influente para selecionar a cor do dente é o gênero. Em um estudo realizado por Alfouzan, Alqahtani e Tashkandi (2017), seus resultados não obtiveram diferenças estatisticamente significantes em relação a capacidade dos indivíduos do sexo masculino e feminino em selecionar a cor. Entretanto, Lehmann et al. (2017), observou que os operadores do sexo feminino apresentaram melhores resultados do que os operadores do sexo masculino.

Alguns autores ainda consideram que o fundo e o que está em volta do paciente, podem afetar a percepção do croma e matiz. Por exemplo, a maquiagem, cor dos lábios, bochechas, língua, gengiva, entre outros, afetam e causam variação no tom dos dentes. O mais aconselhado, portanto, é pedir a remoção da maquiagem do paciente e usar um fundo cinza neutro durante o procedimento (Ferreira, 2020).

\section{Seleção da Resina Composta}

A transmissão da luz no esmalte é cerca de 70\%, o que confere a esse tecido características translúcidas. Já na dentina é de $52,6 \%$, o que a torna mais opaca. Dessa forma, para ter características mais próximas do dente natural, resinas mais transparentes devem ser utilizadas para reproduzir o esmalte, enquanto resinas mais opacas devem ser utilizadas para confeccionar a dentina. Os fabricantes dividem as resinas em resinas específicas para esmalte (E-esmalte, T-translúcido) e para dentina (D-dentina, B-body, O-opaco) e ainda fornecem resinas com cores específicas que permitem a confecção de efeitos especiais como transparência incisal, halo opalescente, bem como a manipulação do valor na restauração (Carvalho et al., 2020; Pereira et al., 2018).

Ao realizar restaurações em dentes anteriores, é importante atentar para as suas três inclinações anatômicas. Divididas em terço cervical, médio e incisal. O terço cervical é a parte mais saturada do dente, pois nessa região existe uma fina espessura de esmalte, o que permite visualizar com mais intensidade a porção escurecida da dentina. Além disso, a área cervical é característica de mais absorção da luz do que reflexão (Carvalho et al., 2020; Pereira et al., 2018). O terço incisal, por sua vez, há mais esmalte do que dentina, logo é um terço mais translúcido, pois transmite mais facilmente a luz. Sendo assim, usam-se resinas mais translúcidas. Finalmente, o terço médio é a área que apresenta uma maior luminosidade, visto que reflete muita luz, não existe a influência do fundo escuro da boca, como tem no terço incisal. Por ser o terço mais plano, ele 
favorece o processo de reflexão da luz, obtendo uma área de maior luminosidade e, portanto, mais esbranquiçada (Lobato et al., 2019; Nahsan et al., 2012).

Ao entender o dinamismo óptico dos tecidos dentários podemos usar diferentes resinas e marcas comerciais que atinjam um determinado objetivo. Desse modo, uma técnica bastante utilizada que permite a utilização de diferentes tipos de resinas, restaurando o esmalte e dentina separadamente, é a abordagem incremental ou técnica de estratificação (Vanini e Mangani, 2001). A estratificação de restaurações compostas para fins estéticos iniciou com o desenvolvimento da fotopolimerização em etapas e com o lançamento de várias resinas compostos, como a Herculite XR (Kerr, Orange-CA, USA, 1985), proporcionando mais opções de tons de materiais para o sistema restaurador (Devoto et al., 2010; Dietschi e Fahl, 2016). Esta técnica incremental permite mascarar adequadamente os limites entre o dente e a restauração, para reproduzir com mais precisão as características ópticas e físico-mecânicas de cada substrato dentário (Carvalho et al., 2020; Correia; Oliveira e Silva, 2005; Lobato et al., 2019; Pereira et al., 2018).

Ao iniciar a restauração de um dente anterior, é necessária a utilização da matriz de poliéster ou guias customizadas. Esta segunda opção apresenta maiores vantagens, pois fornece dimensões harmônicas em procedimentos restauradores, além de ter um maior controle da espessura dos incrementos da resina composta (Gil e Abreu, 2015).

Sendo assim, considerando uma cavidade que atinja a região vestibular e palatina, a primeira camada de resina será para a reconstrução do esmalte palatino/lingual, utilizando uma resina composta translúcida de fina espessura, preenchendo toda a região palatina/lingual do dente (Dietschi e Fahl, 2016; Villarroel et al., 2011). A segunda camada de resina refere-se a dentina. Todavia, a construção da dentina deve ser dividida em duas partes: a dentina interna e dentina externa. Posto que, nos dentes naturais ocorre uma diminuição progressiva de saturação da região cervical para a incisal, bem como da região mais interna para a superfície do dente (Gil e Abreu, 2015; Dietschi e Fahl, 2016; Lobato et al., 2019; Pereira et al., 2018).

Considerando isso, a segunda camada será para reproduzir a dentina localizada mais internamente e direcionada ao terço cervical, o croma deve ser um tom acima do selecionado, para assim atingir o aspecto harmonioso e natural da restauração. Na terceira camada haverá a reprodução da dentina mais superficial/externa, esta é a dentina encontrada mais próxima da junção esmalte-dentina, assim como a dentina encontrada no terço médio e direcionada para o terço incisal. Dessa maneira, faz-se necessário o uso de resinas com propriedades ópticas fluorescentes, delimitando os mamelões (Gaião et al., 2019; Dietschi e Fahl, 2016; Lobato et al., 2019; Pereira et al., 2018).

$\mathrm{Na}$ quarta camada são usadas resinas opacas, para mimetizar o halo opalescente, e translúcido, para reconstruir a translucidez da borda incisal. A penúltima camada será para reconstruir o esmalte vestibular, esmalte de corpo, sendo necessário ser colocado com espessura de $0,5 \mathrm{~mm}$, não devendo ultrapassar $1 \mathrm{~mm}$ para não diminuir o valor final. Essa camada utiliza a resina de esmalte com croma na cor selecionada. Por fim, a sexta e última camada é direcionada para a finalização da confecção da porção incisal do esmalte, usando uma resina de esmalte sem croma para obter translucidez (Nahsan et al., 2012; Gueli et al., 2017). Quando a resina de esmalte é feita com uma alta espessura, a restauração pode obter um aspecto cinza. Portanto, é necessário que a resina de esmalte tenha metade ou menos da metade da espessura do esmalte de um dente natural, aproximadamente 0,5mm de espessura (Dietschi e Fahl, 2016; Ottoboni et al., 2018).

A maior parte dos erros pelos cirurgiões dentistas que ocorrem durante a seleção de cor do dente para uma restauração é decorrente de falhas na escolha do valor, seja por desconsiderar sua importância e por não saber escolher o mesmo ou por usá-lo em incrementos desproporcionais. Há certa dificuldade em diferenciar resinas opacas e resinas translúcidas, pois as marcas comerciais das resinas focam-se no croma e matiz, sem, muitas vezes, mencionar o valor na embalagem (Fondriest, 2017; Hirata, 2017). O valor das resinas é determinado pela capacidade destas refletirem ou absorverem a luz, agindo como objetos translúcidos ou opacos. Então, quando se aplica nas restaurações uma quantidade grande de resina opaca esta vai 
tornar-se mais branca (aspecto leitoso) com um valor mais alto, o mesmo não acontece quando aplica uma grande quantidade de resina translúcida, pois a restauração torna-se acinzentada com um valor mais baixo (Hirata, 2017).

Algumas características se tornam evidentes quando são realizadas restaurações de resina composta em dente anterior, como a percepção de um dente mais largo ou mais curto aparentemente. Isso é provocado devido os dentes naturais possuírem áreas de reflexão e áreas de sombra. A área de reflexão, também conhecida como área plana, é definida geralmente por duas arestas longitudinais e influencia na largura aparente dos dentes, considerada a área do dente que fica em maior evidência e determina o tamanho aparente do dente (Lucena et al., 2016). Quanto mais essas arestas se aproximam do centro da superfície vestibular, menor é a área plana, ou seja, maior vai ser a área de sombra, dando a ilusão de óptica de dente estreito e menos luminoso. Por outro lado, quanto mais elas são deslocadas no sentido das superfícies proximais, maior é a área plana dando a impressão de dente mais largo e mais luminoso, pois reflete mais luz. Sendo assim, quando o intuito é aumentar a luminosidade do dente e deixá-lo com sensação de maior, é necessário aumentar a largura da área plana. Ao contrário, se essa área plana é menor, por consequência a área de sombra se tornará maior, permitindo uma sensação de dente menor e menos luminoso (Cardoso et al., 2006; Lucena et al., 2016).

Por fim, é importante estabelecer um protocolo de acabamento e polimento, para otimizar a forma e a textura dental, além de obter um brilho final adequado. Sendo possível, assim, alcançar um procedimento restaurador eficaz.

\section{Considerações Finais}

O dente natural é policromático, possui uma grande variedade de cores e, por isso, a seleção de cor deve ser realizada da maneira correta para reproduzir artificialmente as características intrínsecas do dente. O dentista deve conhecer e compreender as etapas da seleção de cor, bem como possuir conhecimento aprofundado sobre a cor, luz e das características estruturais dos dentes, a fim de definir as diferentes nuances de cada dente, utilizando as melhores técnicas para dar naturalidade e sucesso estético para o paciente.

Além disso, para economizar tempo na reconstrução do dente é necessário gerenciar a quantidade de compósito aplicada, pois um pequeno excesso ou até mesmo falta de compósito pode determinar falha estética e a necessidade de repetir a restauração. Para isso, há uma variedade de técnicas a serem seguidas para determinar a cor de um dente, que vão desde escalas de cores, testes com incrementos resinosos, à espectrofotómetros. Técnicas que devem ser estudadas e adequadas para atingir um procedimento restaurador com excelente nível estético. Do mesmo modo, deve-se buscar minimizar as influencias que dificultam na determinação da cor dos dentes, causados pelas variáveis da fonte de luz disponível, do objeto, operador, dentre outros. Concomitantemente, a seleção de cor possui etapas que não devem ser negligenciadas, ao contrário, precisam ser seguidas com cuidado e previsibilidade para melhorar a qualidade das restaurações. Portanto, é fundamental a atualização e uma formação mais detalhada em relação à ciência e escolha da cor, devido à complexidade e subjetividade da mesma. Ademais, sugere-se para trabalhos futuros que sejam pesquisados o desenvolvimento de novas alternativas para a seleção de cor dentária, bem como métodos com maior facilidade e rapidez para a escolha de cor.

\section{Referências}

Agrawal, V. \& Kapoor, S. (2013). Color and Shade Management in Esthetic Dentistry. Universal Research Journal of Dentistry, 3 (3), $120-7$.

Amaral, B. R. (2006). Análise de variáveis que podem interferir na seleção de cor dos dentes. Universidade de Brasília, Brasília.

Ayna, B. et al. (2018). Effect of Different Esthetic Post-Core Materials on Color of Direct-Composite Restorations: A Preliminary Clinical Study. Medical science monitor: international medical journal of experimental and clinical research, 24, 4091-4100.

Bahr, M. et al. (2015). Avaliação visual da cor mediante diferentes fontes de luz. Prosthesis Laboratory in Science, 4 (14).

Bhatia, S. et al. (2013). Color and Shade Matching: Significance in Dentistry. Malaysian Dental Journal, 35, 6-11. 
Bottino, M. A., Faria, R., Valandro, L. F. (2009). Percepção - estética em próteses livres de metal em dentes naturais e implantes. São Paulo: Artes Médicas. $804 \mathrm{p}$.

Cardoso, P. C. et al. (2006). Princípios Estéticos para reanatomização de dentes anteriores após tratamento ortodôntico: Rela to Clínico. Clínica. International Journal of Brazilian Dentistry, 2, 32-37.

Carvalho, G. A. O. et al. (2020). Stratification of resin composed with use of silicone barrier: literature review. Research, Society and Development, 9 (7), 246974114.

Chu, S. J. et al. (2010). Dental color matching instruments and systems. Review of clinical and research aspects. J Dent., 38, 2-16.

Costa, A. R. (2018). Implementação Tecnológica Computacional Para Escolha De Cor E Mapeamento Em Restaurações Com Resina Composta. Faculdade de Odontologia da UFU, Uberlândia.

Dietschi, D., Fahl, N. (2016). Shading concepts and layering techniques to master direct anterior composite restorations: an update. Br Dent J., 221 (12), 765 771.

Ferraris, F. et al. (2015). Influence of enamel composite thickness on value, chroma and translucency of a high and a non-high refractive index resin composite. The International Journal of Esthetic Dentistry, 9 (3), 1-21.

Ferreira, E. C. S. (2020). Dificuldade de percepção da cor pelos médicos dentistas. Instituto Universitário Egas Moniz.

Ferreira, F. G. (2017). Sistema smile lite como método auxiliar na escolha de cor em odontologia. Universidade de Santa Cruz do Sul.

Fondriest, J. (2003). Shade Matching in Restorative Dentistry: The Science and Strategies. The International Journal of Periodontics and Restorative Dentistry, 23 (5), 467-479.

Gaião, L. F. C. U. et al. (2019). Clinical Steps for Restoration of Fractured Anterior Teeth: Color Protocol with Non-Vita Scale. Case Reports in Dentistry, v.2019.

Gasparik, C. et al. (2014). Influence of light source and clinical experience on shade matching. Clujul Medical, 87 (1), 30, Romania.

Gasparik, C. et al. (2015). Shade-matching performance using a new light-correcting device. Journal of Esthetic and Restorative Dentistry, 27 (5), $285-292$, Romania.

Gil, A. M. C. \& Abreu, G. (2015). Recomendaciones para el empleo práctico de resinas compuestas en restauraciones estéticas. Rev Cubana Estomatol, Ciudad de La Habana, 52 (3), 293-313.

Gueli, A. M. et al. (2017). Color specification of two new resin composites and influence of stratification on their chromatic perception. Color Res Appl, 42, 684-692.

Hilton, T. J. et al. (2013). Summit's fundamentals of operative dentistry a contemporary approach. 2 (2), 285-299.

Hirata, R. (2017). Shortcuts in esthetic dentistry. 5 (2), 285-299.

Igiel, C. et al. (2016). Dental color matching: A comparison between visual and instrumental methods. Dental Materials Journal, 35 (1), 63-69.

Igiel, C. et al. (2017). Reliability of visual and instrumental color matching. Journal of Esthetic and Restorative Dentistry, 29 (5), 303-308, Germany.

Jain, A., Bhat, V., Hedge, C. (2017). A Study to Analyze the Paramount Way of Shade Selection among Restorative Dentists in South Canara District, Karnataka. NUJHS, 7 (2).

Kalantari, M. H. et al. (2017). Evaluation of accuracy of shade selection using two spectrophotometer systems: Vita Easyshade and Degudent Shadepilot. European Journal Dentistry, 11, 196-200.

Lobato, M. et al. (2019). Reanatomização de dente conóide com resina composta por meio de técnica de aplicação simplificada. Revista Naval de Odontologia, $46(1)$.

Lucena, A. L. R. et al. (2016). Cores em restaurações estéticas: Conceitos e Fundamentos práticos. Odontol. Clín.-Cient., Recife, 15 (4), 235 - 240.

Malainho, L. C. L. (2019). Caracterização da principal metodologia utilizada na determinação de cor dentária. Instituto de Ciências da Saúde, Lisboa, Viseu.

Melo, A. K. V. et al. (2020). Princípios microestéticos a serem considerados durante uma reabilitação oral: Revisão de literatura. Odontol. Clín.-Cient., Recife, 19 (4) $315-321$.

Moodley, D. et al. (2015). Comparison of colour differences in visual versus spectrophotometric shade matching. South African Dental Journal, 70 (9), $402-$ 407.

Nahsan, F. P. S. et al. (2012). Clinical strategies for esthetic excellence in anterior tooth restorations: understanding color and composite resin selection. $J$. Appl. Oral Sci., Bauru, 20 (2), 151-156.

Negahdari, R. et al. (2016). Comparison of Shade Match Compatibility between Vitapan Classical and 3D Master Shade Guide Systems by Dental Students in Tabriz Faculty of Dentistry. Advances in Bioscience and Clinical Medicine, 4 (1), 4-10.

Ottoboni, T. et al. (2018). Resinas compostas em dentes anteriores. The Aesthetics yearbook, 1 (6).

Paolone, G. et al. (2014). Composite shade guides and color matching. The international journal of esthetic dentistry, 9 (2), 164-82. 
Parameswaran, V. et al. (2016). Comparison of accuracies of an intraoral spectrophotometer and conventional visual method for shade matching using two shade guide systems. The Journal of the Indian Prosthodontic Society, 16 (4), 352, India.

Paravina, R. D. et al. (2019). Colorimetric comparison between two shade guides used for visual evaluation of tooth whitening efficacy. Srpski Arhiv Za Celokupno Lekarstvo, 147 (3-4), 142-147.

Paravina, R. D. (2009). Performance assessment of dental shade guides. Journal of Dentistry, Guildford, 37 (1), $15-20$.

Paravina, R. D. et al. (2007). Optimization of tooth color and shade guide design. J Prosthodont, 16 (4), 269-276.

Paravina, R.D., Powers, J.M. \& Fay, R.M. (2002). Color comparison of two shade guides. Int J Prosthodont, 15 (1), $73-8$.

Pereira, A. C. et al. (2018). Estratificação incremental com resina composta: reprodução de efeitos ópticos incisais em restauração classe IV. Ustasalud, 17, 57-66.

Pereira, G. N. (2019). Métodos de seleção de cor. Universidade Federal De Minas Gerais, Belo Horizonte.

Pepelascov, D. E. et al. (2018). Opalescence of restorative materials and optical properties of human tooth - a literature of review. Brazilian Journal of Surgery and Clinical Research, 24 (2), 119-124.

Radaelli, M. T. B. et al. (2013). Propriedades Ópticas Relacionadas à Estética Dental. J Oral Invest, 1 (2), 22-27.

Reus, J. S. (2017). Seleção de Cor em Restaurações Estéticas - Protocolo de Transmissão de Informação Clínica-Laboratório. Instituto Universitário de Ciências da Saúde.

Ribeiro, L. F. A. \& Grajeda, F. M. C. de. (2019). Cor na odontologia restauradora moderna: Revisão de literatura. Revista de Iniciação Científica da Universidade Vale do Rio Verde, Três Corações, 9 (1), 57-68.

Rodrigues, T. P. (2007). Procedimentos de seleção de cor, percepção visual de diferença de cor e fluorescência em Odontologia Estética. Universidade Estadual Paulista, Araraquara.

Schmeling, M. et al. (2012). Translucency of value resin composites used to replace enamel in stratified composite restoration techniques. Jornal Esthet Restor Dentistry, 24 (1), 53-58.

Schmeling, M. et al. (2010). Chromatic Influence of Value Resin Composites. Operative Dentistry, 35 (1), 44-49.

Sikri, V. (2010). Color: Implications in dentistry. Journal Conservative Dentistry, 13 (4), 249.

Śmielecka, M. \& Dorocka-bobkowska, B. (2020). Effects of different light sources on tooth shade selection. Dent Med Probl, 57 (1), $61-66$.

Sousa, S. F. T. (2019). Seleção de Cor - Restaurações Anteriores Diretas. Instituto Universitário de Ciências da Saúde, Gandra.

Tokumi, A. F. S. (2007). Cor em Odontologia. Universidade Federal de Santa Catarina, Florianópolis.

Tsiliagkou, A. et al. (2016). Evaluation of reliability and validity of three dental colour-matching devices. The International Journal Of Esthetic Dentistry, 11 (1).

Valenzuela-aranguiz, V. et al. (2016). Selección de color dentario: comparación de los métodos visual y espectrofotométrico. Rev. Clin. Periodoncia Implantol. Rehabil. Oral, Santiago, 9 (2), 163-167.

Vanini, L. (2011). A cor segundo Lorenzo Vanini. Rev Dental Press Estét, 8 (4), 98-107.

Vanini, L. \& Mangani, F. (2001). Determination and communication of color using the five color dimensions of teeth. Pract Proced Aesthet Dent, 13 (1), 1926.

Villarroel, M. et al. (2011). Direct esthetic restorations based on translucency and opacity of composite resins. J Esthet Restor Dent, 23 (2), $73-87$.

Wanderley e Lima, R. B. et al. (2013). Reabilitação Estética Anterior pela Técnica do Facetamento - Relato de Caso. Revista Brasileira de Ciências da Saúde, 17 (4), 363-370.

Werneck, R. D. et al. (2016). Odontology students efficiency in color selection with Vita 3D-Master shade guide. Full dent. sci, 7 (26), $92-98$. 